\title{
Influencia del té verde y del té de jengibre en el rendimiento deportivo del futbolista
}

Salas-Hernández Alejandro*, Hurtado-Ovalle Silvia Anel*, Marín-Padilla Ana Lorena*, Arriaga-Domínguez Mariana Paulina*, Salas-Herrera María Milagros*, Martín del Campo-Cervantes Judith**, Esparza-Ramos Susana Berenice***

\begin{tabular}{|c|c|}
\hline 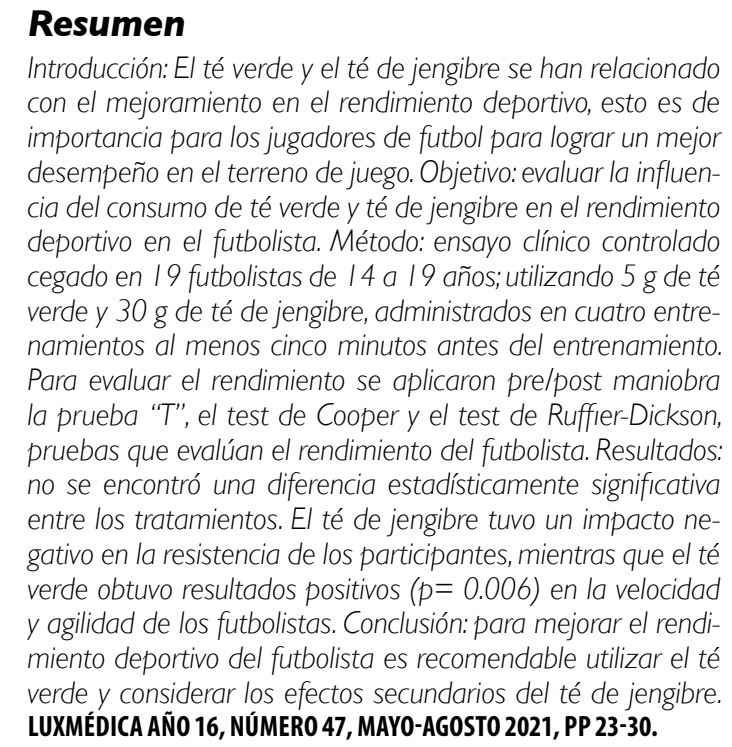 & 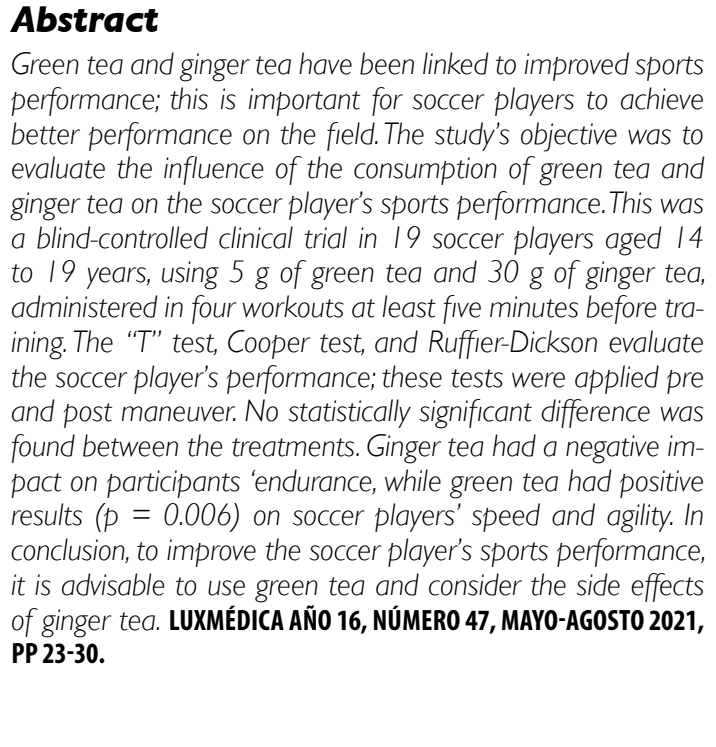 \\
\hline $\begin{array}{r}\text { Palabras clave: té verde, té de jengibre, rendimiento } \\
\text { deportivo, futbolista. }\end{array}$ & $\begin{array}{l}\text { eywords: green tea, ginger tea, sports performance, } \\
\text { ccer player }\end{array}$ \\
\hline
\end{tabular}

* Estudiante de la Licenciatura en Nutrición del Centro Ciencias de la Salud de la Universidad Autónoma de Aguascalientes, Aguascalientes, México. orcid.org/0000-0001-9399-1680. Correo electrónico: ale_x10@outlook.com

* Estudiante de la Licenciatura en Nutrición del Centro Ciencias de la Salud de la Universidad Autónoma de Aguascalientes, Aguascalientes, México. orcid.org/0000-0001-6636-2977. Correo electrónico silvianel1995@hotmail.com

Estudiante de la Licenciatura en Nutrición del Centro Ciencias de la Salud de la Universidad Autónoma de Aguascalientes, Aguascalientes, México. orcid.org/0000-0002-3654-8583. Correo electrónico: analorenamarinpad@outlook.com

* Estudiante de la Licenciatura en Nutrición del Centro Ciencias de la Salud de la Universidad Autónoma de Aguascalientes, Aguascalientes, México. orcid.org/0000-0002-3041-1033. Correo electrónico: mariana.arriaga97@outlook.es

* Estudiante de la Licenciatura en Nutrición del Centro Ciencias de la Salud de la Universidad Autónoma de Aguascalientes, Aguascalientes, México. orcid.org/0000-0002-7017-2700. Correo electrónico: mmsh.9529@gmail.com

** Profesora Investigadora del Departamento de Nutrición y Cultura Física, Centro Ciencias de la Salud de la Universidad Autónoma de Aguascalientes, México. orcid.org/0000-0003-1855-6309. Correo electrónico: jmartic@correo.uaa.mx

*** Profesora Investigadora del Departamento de Medicina del Centro Ciencias de la Salud de la Universidad Autónoma de Aguascalientes. México. orcid.org/0000-0002-7388-3305 Correo electrónico: dra.esparza.ramos@hotmail.com

Fecha de recibido: 9 de junio 2020

Fecha de aceptación: 3 de diciembre 2020

Correspondencia: Judith Martín del Campo Cervantes PhD. Departamento de Nutrición y Cultura Física Centro de Ciencias de la Salud. Edificio 107, Ciudad Universitaria. Universidad Autónoma de Aguascalientes. Av. Aguascalientes No. 904, Aguascalientes, Ags. México CP 20131. Tel: +52 (449) 9107400 ext. 53355. Correo electrónico: jmartic@correo.uaa.mx 


\section{Introducción}

El rendimiento deportivo se define como "un proceso que es el resultado de una acción, como aprovechamiento máximo de una ejecución, para poder realizar una tarea lo mejor posible, donde influyen factores de aprendizaje como la experiencia, la atención, la disposición al esfuerzo, el talento, la creatividad, la preparación psicológica, etc.". Este aspecto es de gran importancia para que los jugadores de futbol puedan desempeñarse en el terreno de juego, por lo que se buscan alimentos funcionales que puedan ayudar a mejorarlo. ${ }^{1}$

Durante los entrenamientos o competencias se genera daño muscular inducido por el ejercicio como inflamación, dolor, fatiga, cansancio, compromiso de la función muscular, entre otros efectos negativos que ocasionan un mayor periodo de recuperación entre entrenamientos o competencias. ${ }^{2}$ Este estrés oxidativo se relaciona con la fatiga muscular, la creación de radicales libres, de especies reactivas de oxígeno y en general al proceso de óxido-reducción que en el músculo ocasiona daño en la fibra muscular y fatiga. ${ }^{3}$

Lo anterior ha ocasionado el estudio de diversas técnicas y productos para tratar de disminuir dicho daño y mejorar el proceso de recuperación. ${ }^{2}$ El uso de plantas cada vez más ha sido aplicado en el deporte por los beneficios que pueden tener los compuestos activos sobre el rendimiento del atleta, no sólo sobre el proceso de oxidación, sino también el proceso inflamatorio, la resistencia y el rendimiento físico. ${ }^{3}$

Se ha identificado que las hierbas y plantas tienen compuestos bioactivos como polifenoles, terpenoides y alcaloides que generan un efecto fisiológico en el cuerpo humano que pueden tener beneficios sobre parámetros deportivos como mejoradores del rendimiento físico, fuerza muscular, masa muscular, cambios en la composición corporal, entre otros. ${ }^{4}$

Los compuestos activos de las plantas o hierbas tienen propiedades antioxidantes sobre el daño oxidativo y la disminución de radicales libres; lo anterior puede disminuir el estrés oxidativo inducido por el ejercicio y mejorar la recuperación de los atletas. ${ }^{4}$

Los alimentos funcionales son aquellos en los que está demostrado la generación de un efecto benéfico sobre el organismo; algunos de los alimentos que entran en este grupo son el té verde y el té jengibre. ${ }^{5,6}$ El té verde (Camellia sinensis) es considerado un alimento funcional por sus efectos cardioprotectores, ya que actúa sobre el metabolismo de los lípidos y tiene un efecto vasoconstrictor $^{7-11}$. Es un estimulador del sistema nervioso y potente antioxidante por su contenido de polifenoles ${ }^{2}$; se ha encontrado que aumenta el rendimiento, la oxidación lipídica muscular y reduce el daño oxidativo del ADN inducido por el ejercicio. ${ }^{4}$ Además de esto, varios estudios hablan de influencia sobre el rendimiento deportivo debido a su contenido de cafeína, antioxidantes y flavonoles. Una taza de té verde, hecho con hojas de té verde naturales, contie- 
ne alrededor de $41 \mathrm{mg}$ de cafeína. La toma de una taza de este té al día no es considerada como dopaje por la Agencia Mundial Anti-Doping (World Anti-Doping Agency. WADA) ni por el Comité Olímpico Internacional (COI). ${ }^{4-8}$

El jengibre (Zingiberofficinale Roscoe, Zingiberaceae) ${ }^{4}$ también es considerado alimento funcional; es utilizado en el tratamiento de enfermedades degenerativas como la artritis y el reumatismo, como protector en el tracto gastrointestinal, en trastornos cardiovasculares y cerebrovasculares, diabetes y como auxiliar en el tratamiento del cáncer gracias a su contenido de polifenoles. También se le atribuyen propiedades antimicrobianas, antiinflamatorias y antioxidantes; éstas últimas son debidas a su contenido de gingeroles y 6-shogaol, moléculas bioactivas del jengibre que colaboran en la reducción del estrés oxidativo al eliminar los radicales libres producidos en los sistemas biológicos del organismo. Su efecto antiinflamatorio es muy importante ya que inhibe la síntesis de prostaglandinas y leucotrienos por lo que ayuda al tratamiento de diversos padecimientos. Este efecto se atribuye a los componentes bioactivos del jengibre. ${ }^{12-14}$

Tiene efectos antiinflamatorios comprobados lo cual puede traducirse en una disminución en la sensación de fatiga de los atletas ${ }^{4}$ y en la mejora del rendimiento deportivo, ya que contribuyen a la disminución del dolor muscular durante y después del ejercicio. De acuerdo con investigaciones en roedores, el jengibre tiene propiedades analgésicas similares a los antiinflamatorios no esteroideos (AINES)..$^{15}$ El jengibre puede disminuir la sensación de dolor muscular debido a sus efectos analgésicos al modular la síntesis de prostaglandinas, lo que puede incrementar el rendimiento y resistencia del atleta. ${ }^{2}$

Sin embargo, el consumo de estos alimentos por sí solos no generan un efecto positivo en el rendimiento deportivo si no se tiene un descanso adecuado, una buena alimentación con las características necesarias para un atleta, el uso de suplementos alimenticios, así como distintos métodos para tener una recuperación post-ejercicio más satisfactoria. ${ }^{7}$

El propósito de esta investigación fue evaluar la influencia del consumo de té verde y té de jengibre antes del entrenamiento y su efecto en el rendimiento deportivo en el futbolista de 14 a 19 años.

\section{Material y métodos}

Se realizó un ensayo clínico controlado cegado en 19 futbolistas varones de 14 a 19 años de la Escuela de futbol Rayados de Monterrey Aguascalientes. Se excluyeron futbolistas que tuvieran el hábito del tabaquismo, presentaran enfermedades car- diovasculares, respiratorias y diabetes; se eliminaron futbolistas que se lesionaron durante el entrenamiento para no afectar los resultados. Materiales: té verde, té de jengibre, cronómetro digital marca Westward precisión $\pm 0.01 \mathrm{seg}$, flexómetro Stanley Pro-modelo 30088 precisión de $25 \mathrm{~mm}$, 
silbato y conos de plástico. Maniobra: el jengibre se administró a los futbolistas mediante un té, el cual fue servido en vasos de $250 \mathrm{ml}$ con $30 \mathrm{~g}$ de jengibre o con $5 \mathrm{~g}$ de hojas de té verde natural; ambos tés se consumieron al menos cinco minutos antes del entrenamiento. El estudio fue cegado debido a que los futbolistas desconocían el tipo de té que se les proporcionó. Se cuidó que las infusiones de té fueran estandarizadas para cada toma y para cada futbolista. Se realizaron mediciones de rendimiento físico (prueba T, Test de Ruffier-Dickson y test de Cooper) pre - post consumo del té verde o de jengibre en 4 sesiones de entrenamiento; se cuidaron que las condiciones climáticas fueran las mismas en los 4 entrenamientos, así como también se les pidió a los participantes que no modificaran su comportamiento de sueño, actividades durante el día y alimentación para mantener condiciones equiparables para cada entrenamiento.

\section{Métodos}

Prueba T: esta prueba evalúa la agilidad y rapidez del futbolista. Para aplicarla se colocaron conos plásticos en forma de T en el piso, cada línea con una longitud de nueve metros. El individuo recorrió la $T$ a toda velocidad desplazándose de frente, lateralmente y hacia atrás sin cruzar los pies en ningún momento, se tomó el tiempo con cronómetro. La prueba se realizó tres veces y el resultado utilizado fue el mejor tiempo obtenido. ${ }^{16}$

Test de Ruffier-Dickson: utilizada para evaluar la frecuencia cardiaca (FC) y la recuperación del futbolista. Para la toma de la FC se colocaron el dedo índice y el dedo medio del investigador en el lado derecho del cuello del participante, se localizó el pulso y se contaron las pulsaciones por 30 segundos, al terminar la cuenta se multiplicó por dos para obtener los latidos por minuto (lpm). ${ }^{17}$ Para el test se tomó la FC en reposo de los individuos (pulso 0), posteriormente se realizaron sentadillas durante 45 segundos e inmediatamente se tomó la FC (pulso 1), se descansó por un minuto y se volvió a tomar la FC al terminar el descanso (pulso 2). El resultado se calculó con la ecuación para determinar el índice de Ruffier-Dickson:18 ((Pulso 0 + pulso $1+$ pulso 2)-200)/100.

Test de Cooper: utilizado para evaluar la resistencia física del futbolista. El individuo corrió durante 12 minutos en una superficie plana, al terminar el tiempo se les indicó no caminar hacia adelante o hacia atrás para no aumentar o disminuir la distancia recorrida, se midió la distancia con un flexómetro y, después de cinco minutos de terminar el tiempo de la prueba, se midió la FC. ${ }^{19}$

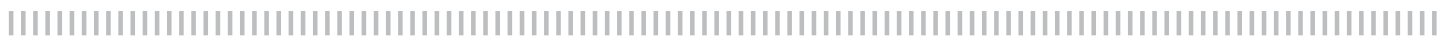

\section{Resultados}

Tras la toma del té de jengibre se observaron un aumento de 12.75 latidos por minuto ( $p$ $=0.033$ ) y de una disminución de 209.4 metros recorridos $(p=0.017)$ en el test de Cooper, lo que indica cambios desfavorables en cuanto a la resistencia de los jugadores y a la distancia recorrida. Los futbolistas evaluados normalizaron su frecuencia cardíaca con resultados de "satisfactorio" y "bien", con respecto a su resistencia física. Mientras que con el té verde se observó una diferencia significativa en la prueba T ( $p=0.006)$, debido a que los tiempos de realización de la prueba fueron menores en comparación con los resultados pre-prueba (Tabla 1). En el resto de las pruebas no se encontraron diferencias estadísticamente significativas. 


\section{Tabla I}

Comparación de las pruebas de rendimiento deportivo antes y después de la toma de té verde y té de jengibre.

\begin{tabular}{|l|c|c|c|c|c|c|}
\hline & \multicolumn{3}{c}{ Té de jengibre } & \multicolumn{3}{c|}{ Té verde } \\
\hline Pruebas & $\begin{array}{c}\text { Antes } \\
\text { (media, DE) }\end{array}$ & $\begin{array}{c}\text { Después (me- } \\
\text { dia, DE) }\end{array}$ & $p$ & $\begin{array}{c}\text { Antes } \\
\text { (media, DE) }\end{array}$ & $\begin{array}{c}\text { Después (me- } \\
\text { dia, DE) }\end{array}$ & $p$ \\
\hline $\begin{array}{l}\text { Test de Cooper } \\
\text { FC (Imp) }\end{array}$ & $93.75 \pm 15.80$ & $106.50 \pm 17.13$ & $\mathbf{0 . 0 3 3 ^ { b }}$ & $100.00 \pm 18.85$ & $103.82 \pm 18.79$ & $0^{\text {b }}$ \\
\hline $\begin{array}{l}\text { Test de Cooper } \\
\text { Distancia (m) }\end{array}$ & $2377.45 \pm 232.99$ & $2168.05 \pm 301.65$ & $\mathbf{0 . 0 1 7 ^ { \mathrm { b } }}$ & $2284 \pm 310.51$ & $2118.74 \pm 266.81$ & $0.248^{\mathrm{a}}$ \\
\hline $\begin{array}{l}\text { Ruffier-Dickson } \\
\text { (IRD) }\end{array}$ & $8.53 \pm 1.17$ & $8.35 \pm 2.97$ & $0.868^{\mathrm{b}}$ & $10.53 \pm 2.34$ & $9.42 \pm 2.82$ & $0.324^{\mathrm{b}}$ \\
\hline Prueba T (seg) & $10.73 \pm 0.066$ & $9.98 \pm 0.55$ & $0.93^{\mathrm{a}}$ & $11.06 \pm 1.25$ & $10.55 \pm 1.01$ & $\mathbf{0 . 0 0 6}^{\mathrm{a}}$ \\
\hline
\end{tabular}

FC: frecuencia cardiaca, lpm: latidos por minuto, m: metros, IRD: índice de Ruffier-Dickson, Seg:segundos. ${ }^{a}$ Se aplicó el test de Wilcoxon, considerando una significancia estadística $<0.05$.

${ }^{b}$ Se aplicó una prueba $T$ de Student pareada con una significancia estadística $<0.0$

Los participantes comenzaron el estudio con las mismas características en cuanto al rendimiento físico que se les exige en el entrenamiento y se les pidió mantener las mismas condiciones fisiológicas (descanso, alimentación e hidratación) entre los entre- namientos. Al comparar si el consumo de uno u otro té tuvo un mejor parámetro de rendimiento, no se encontraron diferencias significativas entre los resultados de las pruebas aplicadas $(p>0.05)$ como se muestra en la tabla 2.

\section{Tabla 2}

Comparación de la efectividad del té verde contra el té de jengibre en las pruebas de rendimiento deportivo

\begin{tabular}{|l|c|c|c|}
\hline Pruebas & \multicolumn{1}{c}{$\begin{array}{c}\text { Tratamiento } \\
\text { Té de jengibre } \\
\text { (media, DE) }\end{array}$} & $\begin{array}{c}\text { Té verde } \\
\text { (media, DE) }\end{array}$ & $p$ \\
\hline Test de Cooper FC (Ipm) & $106.50 \pm 17.13$ & $103.82 \pm 18.79$ & $0.754^{\mathrm{a}}$ \\
\hline $\begin{array}{l}\text { Test de Cooper } \\
\text { Distancia (m) }\end{array}$ & $2186.05 \pm 301.65$ & $2181.75 \pm 266.81$ & $0.918^{\mathrm{a}}$ \\
\hline Ruffier-Dickson (IRD) & $8.35 \pm 2.97$ & $9.42 \pm 2.82$ & $0.436^{\mathrm{a}}$ \\
\hline Prueba T (s) & $9.99 \pm 5.6$ & $10.55 \pm 1.01$ & $0.173^{\mathrm{a}}$ \\
\hline
\end{tabular}

FC: frecuencia cardiaca, lmp: latidos por minuto, m: metros, IRD: índice de Ruffier-Dickson, s: segundos.

${ }^{a}$ Se utilizó la prueba t de Student no pareada con una significancia estadística $<0.05$. 
Discusión

Matsumara y cols. ${ }^{20}$ en su estudio atribuyen al jengibre la aceleración en la recuperación de la fuerza muscular después del ejercicio intenso. Wilson ${ }^{21}$ también habla de las propiedades antiinflamatorias del jengibre, el cual es comúnmente utilizado por los atletas para prevenir el dolor muscular después del ejercicio, retardando la aparición del cansancio, ayudando a la resistencia y la recuperación del deportista; estos resultados son contrarios a lo encontrado en esta investigación, debido a que los participantes mostraron una disminución en la distancia recorrida y un aumento en la frecuencia cardiaca, ambos aspectos nos indican que en nuestra población el té de jengibre tuvo efectos negativos sobre el rendimiento deportivo; este resultado se pudo deber a la concentración de té de jengibre utilizada en este estudio o la forma de presentación del té, mismos que fueron aspectos que comentaron los futbolistas siendo el mal sabor y los trastornos gastrointestinales los más reportados. Por otra parte, Wilson ${ }^{21}$ menciona los efectos secundarios que produce el jengibre, destacando los problemas gastrointestinales como ardor, acidez y dolor; dichos síntomas fueron presentados por algunos de los participantes después de beber el té, al grado de afectar su rendimiento deportivo. ${ }^{21}$

García $^{22}$ utilizó cápsulas de cafeína $(6 \mathrm{mg} / \mathrm{kg})$ y un placebo para evaluar la mejora del rendimiento del futbolista durante un partido, debido a que el té verde contiene una pequeña cantidad de cafeína se evaluó este aspecto en nuestros participantes. Los resultados de García ${ }^{22}$ muestran que no hubo diferencias significativas entre las distancias recorridas con los diferentes tratamientos, lo cual es compatible con lo demostrado acerca del té verde en nuestro estudio. La dosis utilizada en el presente estudio no provoca ningún efecto positivo hacia la mejora de las distancias recorridas, pues es menor a la utilizada por García. ${ }^{22}$
Sin embargo Cotán y cols., ${ }^{23}$ mencionan que la cafeína tiene efectos positivos en la velocidad y distancia recorrida durante un partido; este resultado sí se presentó en los futbolistas, lo cual puede indicar que la cantidad de cafeína incluida en el té verde pudo haber ocasionado estos resultados.

En nuestra población se encontraron resultados favorables en cuanto a agilidad y rapidez, lo cual se atribuye a las propiedades del té verde. Albert Pérez y cols. ${ }^{24}$ detallan en su estudio que el consumo de té verde mejora de manera significativa el rendimiento de los jugadores de futbol; debido a que el té verde funciona como antiinflamatorio, retardando los procesos de fatiga en el músculo, mejorando el rendimiento deportivo.

Pérez y cols. ${ }^{25}$ demostraron que la resistencia deportiva de los futbolistas se puede mejorar antes, durante y después de la temporada, la mejora puede depender de los ejercicios que se realicen, el entrenamiento neuromuscular, los juegos de balón en espacios reducidos o los métodos de entrenamiento que incluyen velocidad, agilidad y rapidez.

El uso de suplementos y productos herbolarios en el deporte ha ido aumentado en los últimos años con diversas finalidades como lo es el mejoramiento de la masa y fuerza muscular, el rendimiento físico, el efecto analgésico en la recuperación, entre otros efectos ${ }^{4}$, por lo que se han estado realizando diversas investigaciones con diferentes dosis para evaluar el uso seguro de estos compuestos en el deporte.

Tanto el jengibre como el té verde son plantas que por sus compuestos pueden beneficiar en el proceso de recuperación de cualquier atleta; las dosis son variadas y dependen también de la tolerancia de cada persona a los efectos que puedan tener la forma en la que se consumen dichos productos. Debido a que cualquier tipo de ejercicio genera un daño muscular, es importante buscar estrategias que ayuden al atleta a disminuir los efectos deletéreos de 
un entrenamiento o una competencia, con la finalidad de buscar un mejor desempeño y rendimiento físico; dichas estrategias deben ser acordadas por los atletas, los entrenadores y personal de salud como los nutriólogos.

La mejora en el rendimiento deportivo presentada por los jugadores de futbol pudo haber sido influenciada por la toma del té verde y té de jengibre; sin embargo, también es una posibilidad el efecto de los diferentes métodos y frecuencia del entrenamiento que tuvieron los futbolistas.

Una de las limitaciones del estudio fue el tamaño de la muestra, para futuras in- vestigaciones se recomienda ampliarla; así como el uso de un grupo control. La dosis de té de jengibre provocó efectos secundarios en los participantes, por lo que se necesita replantear la dosis a utilizar en siguientes investigaciones o también el buscar diluir la infusión para mejorar el sabor. También se recomienda considerar el tiempo entre la toma del té y la medición de las pruebas físicas finales, para que éstas se realicen con tiempos estándar de recuperación y que se realicen inmediatamente después de dicho periodo.

\section{Conclusiones}

El té de jengibre tiene una influencia negativa en el rendimiento deportivo en cuanto a resistencia y FC, mientras que el té verde tuvo una influencia positiva al mejorar la agilidad y velocidad en los futbolistas de este estudio.

\section{Bibliografía}

1. Pacheco M, Gómez J. Características psicológicas y rendimiento deportivo. Un estudio en jugadores bolivianos de fútbol profesional. Ajayu [online]. 2005;3 (2): 42-67.

2. Ahmed I, Michael H, Evlampia P, Lynn P, Panagiotis K. Resistance Training, Antioxidant Status, and Antioxidant Supplementation. Int J Sport Nutr Exerc Metab. 2019 Sep 1;29(5):539-547. doi: 10.1123/ijsnem.2018-0339.

3. Harty, P.S., Cottet, M.L., Malloy, J.K. et al. Nutritional and Supplementation Strategies to Prevent and Attenuate Exercise-Induced Muscle Damage: a Brief Review. Sports Med - Open 5, 1 (2019). https://doi.org/10.1186/ s40798-018-0176-6

4. Sellami $M$, Slimeni O, Pokrywka A, Kuva?i? G, D Hayes $L$, Milic $M$, Padulo J. Herbal medicine for sports: a review. J Int Soc Sports Nutr. 2018 Mar 15;15:14. doi: 10.1186/s12970-018-0218-y. PMID: 29568244; PMCID: PMC5856322.

5. Martirosyand $D$, Singh J. A new definition of functional food by FFC: what makes a new definition unique? Functional Foods in Health and Disease. Functional Foods in Health and Disease 2015; 5(6):209-223.

6. Valderrábanos OLE, Guzmán VCR, Huerta HRE, Lagunas GJG, Garza ER. Alimentos Funcionales en Pediatría. Alerg Asma Inmunol Pediatr 2014; 23 (3): 47-64.

7. Valenzuela A. El consumo té y la salud: características y propiedades benéficas de esta bebida milenaria. Rev Chil Nutr. 2004; 31 (2): 72-82.
8. Pardo R, Álvarez Y, Barral D, Farry M. Cafeína: un nutriente, un fármaco, o una droga de abuso. Adicciones [revista de internet]. 2007. 19 (3): 225-238.

9. Gallego J, Collado P, Verdu J. Nutrición en el deporte. Ayudas ergo génicas y dopaje. $1^{\text {a }}$ ed. España: editorial Díaz de Santos; 2006.

10. World Anti-Doping Agency. Prohibited list. 2018. Publicado por The World Anti-Doping International Standard. [citado en 2018]. Disponible en: https://www.wada-ama. org/sites/default/files/prohibited_list_2018_en.pdf

11. Howard M, Richard. IOC Anti-Dopind Rules applicable to the games of the winter youth Olympic Games Lillehammer 2016. 2015. International Olympic Comiteé. [citado en 2018]. Disponible en: https:// stillmed.olympic.org/media/Document\% 20Library/ OlympicOrg/Games/YOG/Winter-YOG/YOG-LiIlehammer-2016-Winter-Youth-Olympic-Games/ Anti-Doping-Rules-Circular-Letter-Lillehammer-2016. pdf\# ga $=2.268932597 .541263366 .1528394448$ 1719639767.1528394448

12. Haniadka R, Saldanha E, Sunita V, Palatty PL, Fayadd $R$, Shrinath-Baliga $M$. A review of the gastroprotective effects of ginger (Zingiber officinale Roscoe). Food Funct. 2013;4(6):845-55.

13. Mashhadi N., Ghiasvand R, Askari G, Hariri M, Darvishi $L$, Mofid $M$ et al. Anti-Oxidative and Anti-Inflammatory Effects of Ginger in Health and Physical Activity: Review of Current Evidence. Int J Prev Med. 2013; 4(Suppl 1): S36-S42. 
14. Kennedy D. Polyphenols and the Human Brain: Plant "Secondary Metabolite" Ecologic Roles and Endogenous Signaling Functions Drive Benefits. Adv Nutr. 2014;5(5):51533.

15. Black C, Herring M, Hurley D, O'Connor P. Ginger (Zingiber officinale) Reduces Muscle Pain Caused by Eccentric Exercise. J Pain. 2010;11(9):894-903.

16. Runfitners. [Sede Web] 2017 [citado en 2017] Test de Velocidad y Agilidad para Corredores: El Test T. [Aprox. 7 pantallas] Disponible en: https://runfitners.com/test-develocidad-y-agilidad-para-corredores-el-test-t/

17. Maud P. Foster C. Physiological Assessment of Human Fitness. $2^{\circ}$ edición. Champaign, Illinois: Human Kinetics Book; 2006. pp. 39-62.

18. Blog del Dpto. de educación física. [Sede Web] 2016 [citado en 2017]. Test Ruffier-Dickson. [1 pantalla] Disponible en: https://vallevaldebernardoef.wordpress.com/about/ test-ruffier-dickson/

19. Paleo Training [Sede Web]. España. [citado en 2017]. Tests de Cooper y de 5000m. [Aprox. 3 pantallas] Disponible en: http://www.changewithpaleo.com/test-cooper

20. Matsumara MD, Zavorsky GS, Smoliga JM. The Effects of Pre-exercise Ginger Supplementation on Muscle damage and Delayed Onset Muscle Soreness. Phytother Res. 2015 Jun;29(6):887-893.

21. Wilson PB. Ginger (zingiber officinale) as an analgesic and ergogenic aid in sport: a systemic review. J Strength Cond Res. 2015; 29(10):2980-2995.

22. García A. La cafeína y su efecto ergogénico en el deporte (primera parte). Arch Med Deporte 2016;33(3):200-206.

23. Cotán Cid J D, Fernández de la Fuente A, Mata Ordoñez F, Sánchez Olive A J. Análisis de la composición corporal y del consumo de alimentos y suplementos nutricionales en jugadores de división de honor juvenil de fútbol. Revista EmásF. [Revista de internet]. 2017; 47: 106-126.

24. Albert-Pérez EJ, García-Galbis MR. Efectos del té verde en el estado nutricional; revisión sistemática. Nutr. Hosp. [online]. 2015; 32(4):1417-1431.

25. Pérez J, Martín JP, Vivas JC, Alcaraz PE. Entrenamiento de agilidad en futbolistas: una revisión sistemática. Cultura, Ciencia y Deporte [revista de internet]. 2017; 12(35):127134. 